\title{
The efficacy and safety of naproxen in acute rheumatic fever: The comparative results of 11-year experience with acetylsalicylic acid and naproxen
}

\author{
İbrahim İlker Çetin, Filiz Ekici, Abdullah Kocabaş, Berna Şaylan Çevik, Sancar Eminoğlu, \\ Emine Azak, Ayşe Esin Kibar, Mehmet Emre Arı, Murat Sürücü, Ali Orgun \\ Ankara Children's Hematology Oncology Training and Research Hospital, Pediatric Cardiology Clinic, Ankara, Turkey. \\ E-mail: iicetin@hotmail.com \\ Received: 4th October 2016, Revised: 13th January 2017, Accepted: 26th January 2017
}

\begin{abstract}
SUMMARY: Çetin İ̈, Ekici F, Kocabaş A, Çevik BŞ, Eminoğlu S, Azak E, Kibar AE, Arı ME, Sürücü M, Orgun A. The efficacy and safety of naproxen in acute rheumatic fever: The comparative results of 11-year experience with acetylsalicylic acid and naproxen. Turk J Pediatr 2016; 58: 473-479.

The objective was to compare the efficacy and safety of naproxen (NXN) to acetylsalicylic acid (ASA) in the treatment of acute rheumatic fever (ARF). The data of 338 children were retrospectively analyzed. The patients were grouped according to joint and valve involvement and also drug chosen [methyl prednisolone (mPSL), ASA or NXN]. The treatment results and adverse events in each group were compared. The mean age was 10.3 years and the median follow-up was 62 months. Median time for normalization of acute phase reactants was 1 week in patients given steroids and 2 weeks in patients given ASA or NXN. ASA was replaced with NXN in 18 patients $(10.2 \%)$ due to hepatic toxicity. The rate of rebound, recurrence and the prevalence of rheumatic valve disease were not different in patients given NXN, ASA or mPSL. In conclusion, NXN is a safe and effective alternative to ASA in the treatment of ARF in children.
\end{abstract}

Key words: acute rheumatic fever, acetylsalicylic acid, naproxen, adverse events, efficacy.

Acute rheumatic fever (ARF) is an acute disease of childhood characterized by clinical and laboratory features of systemic inflammation. Although the incidence of ARF has declined in developed countries, it remains high in developing countries and also in poorly resourced communities of developed countries ${ }^{1,2}$.

In mild cases, treatment with acetylsalicylic acid (ASA) is mostly effective in resolving inflammation. In moderate and severe cases steroids together with non-steroidal antiinflammatory drugs (NSAIDs) are required to resolve inflammation. The typical steroid is methyl prednisolone (mPSL) and ASA is the most commonly used NSAID in the treatment of ARF with a dramatic response and resolution of fever and arthritis in 1 to 3 days. However, there is no documented evidence showing superior efficacy of any drug (steroids or NSAIDs) in reducing the risk of rheumatic valve disease (RVD) in patients with $\mathrm{ARF}^{3}$.
The successful use of NSAIDs other than ASA has been reported in the treatment of other childhood rheumatic diseases ${ }^{4-6}$. However, such studies are lacking for the treatment of ARF. There are a few reports showing good results with naproxen (NXN) and tolmetin ${ }^{7-9}$. So, we are in need of new randomized-controlled trials or well-designed cohort studies addressing other anti-inflammatory agents to establish an evidence for the efficacy of these drugs in the treatment of $\mathrm{ARF}^{3}$.

This is a retrospective study and the objective of this study was to compare the clinical efficacy and safety of NXN to ASA in the treatment of ARF. The reduction in inflammation, frequency of adverse drug reactions and rebound rates were compared. This study would add a lot to our practice to establish new insights in that subject. 


\section{Material and Methods}

We retrospectively reviewed the data of patients diagnosed with new onset ARF according to the revised Jones criteria ${ }^{10}$ between January 2005 and December 2015, from a central database. Patients presenting with recurrent attacks or chorea, patients with other accompanying vasculitic or inflammatory conditions [familial Mediterranean fever (FMF), juvenile idiopathic arthritis (JIA) or Henoch-Schoenlein purpura (HSP)] and patients in whom ASA was replaced with NXN due to hepatic toxicity during follow-up were excluded. The presence of isolated mild (silent) carditis was accepted as a major criterion, according to a recent guideline by the American Heart Association (AHA) acknowledging the role of Doppler echocardiography for defining subclinical carditis in $\mathrm{ARF}^{11}$.

The study cohort was divided into 4 subgroups on the basis of joint and valve involvement and also drug chosen for treatment. Group 1 consisted of patients with isolated arthritis, arthritis + mild carditis or isolated mild carditis treated with NXN (1A) or ASA (1B). Group 2 consisted of patients with moderate or severe carditis with or without arthritis treated with mPSL + NXN (2A) or mPSL + ASA (2B) (Fig. 1). This subdivision was made because moderate and severe carditis are typically treated with steroids in addition to NSAIDs.

The presence of valvular insufficiency, pericardial

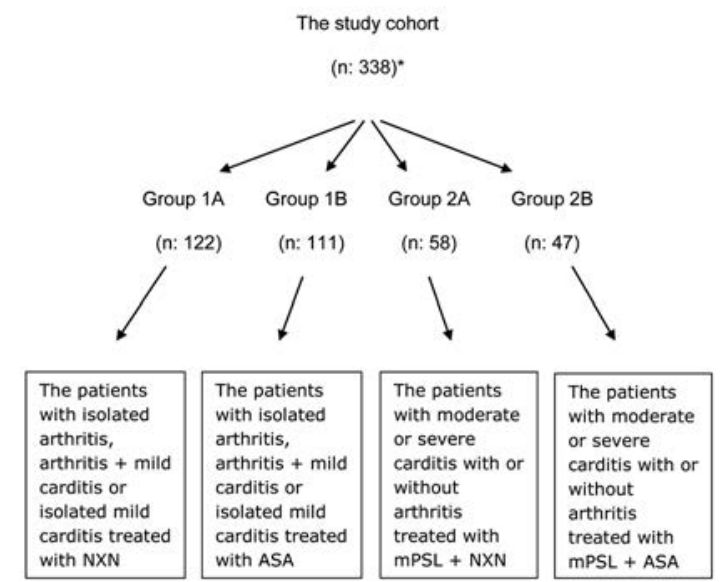

ASA: acetylsalicylic acid, mPSL: methyl prednisolone, NXN: naproxen, *After exclusion of 13 patients diagnosed with other rheumatoid diseases and 18 patients in whom ASA was replaced with NXN due to hepatic toxicity during follow-up.

Fig. 1. The Patient Groups Included in The Study Cohort effusion or myocardial involvement was assessed using conventional echocardiography. Valvular involvement was diagnosed according to previously defined criteria ${ }^{12,13}$. The mild carditis was defined as cardiac involvement with grade 1 valvular regurgitations, moderate carditis was defined as hemodynamically important cardiac involvement with grade 2 valvular regurgitations and severe carditis was defined as hemodynamically important cardiac involvement together with heart failure with grade 3 or 4 valvular regurgitations. In cases with grade 2 valvular regurgitations at 2 or more valves the level of carditis was also defined as severe ${ }^{13}$.

The NSAID used for treatment was selected in an arbitrary manner with increasing frequency of NXN over the last 7 years of the study. Patients in group 1 received NXN orally 15-20 $\mathrm{mg} / \mathrm{kg} / \mathrm{d}(\max 1.5 \mathrm{~g}$ ) in 2-3 divided doses or ASA orally $80-100 \mathrm{mg} / \mathrm{kg} / \mathrm{d}(\max 3.5 \mathrm{~g})$ in 3-4 divided doses. Patients in group 2 received mPSL orally $2 \mathrm{mg} / \mathrm{kg} / \mathrm{d}$ (max $60 \mathrm{mg}$ ) in 3-4 divided doses and when steroid treatment was tapered to minimum anti-inflammatory dose $(1 \mathrm{mg} / \mathrm{kg} / \mathrm{d})$ they were taken to NXN or ASA subgroups.

Drugs were administered until normalization of erythrocyte sedimentation rate (ESR) or C-reactive protein (CRP) in all patients. After normalization of acute phase reactants (APR), the dose of mPSL was tapered over 2 weeks according to therapy duration and the dose of ASA was tapered in 2 weeks. However, tapering was not intended in patients receiving NXN. In all patients, therapy was maintained at least for 4 weeks. The rebound was defined as return of the disease within 4-6 weeks after its apparent cessation while tapering or after termination of drugs. The recurrence was defined as a new episode of the disease occuring after 8 weeks following termination of treatment ${ }^{14}$. Not only neuromotor symptoms were considered, neuropsychiatric symptoms were also taken in to account to diagnose Sydenham's chorea ${ }^{15}$.

The data of patients regarding both symptoms and findings were recorded to a database during treatment process. Patients were asked about the compliance to prophylaxis with benzathine penicillin $G$ and this information is also regularly recorded. All patients were controlled weekly for complete blood count, 

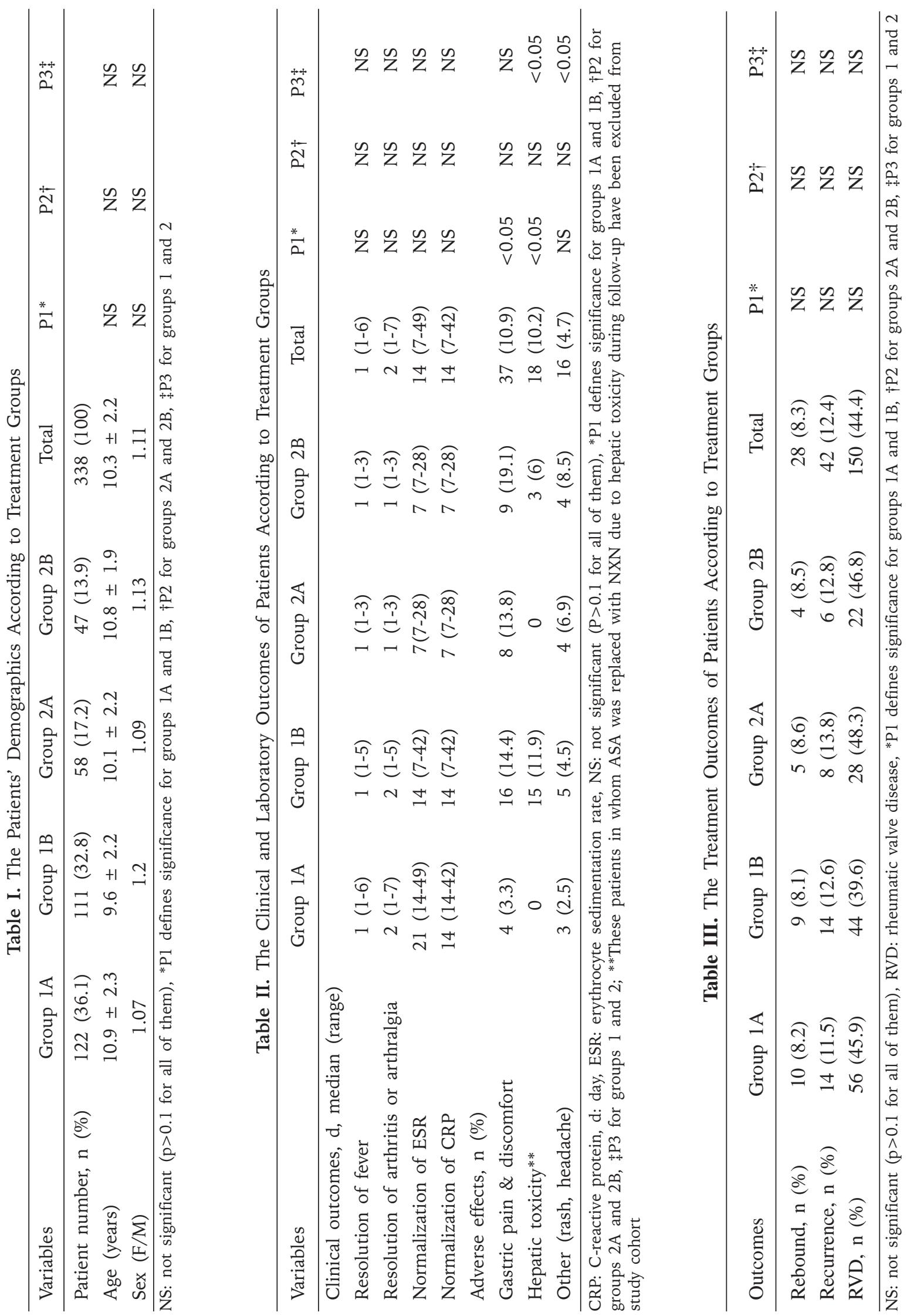
ESR, CRP and echocardiographic examination. The biochemical (Hepatic and renal) and blood coagulation tests were also examined weekly. ASA was decreased to minimum antiinflammatory dose $(50 \mathrm{mg} / \mathrm{kg} / \mathrm{d})$ when serum hepatic enzyme levels exceeded $100 \mathrm{U} / \mathrm{L}$; two-fold the normal levels. Besides, ASA was replaced with NXN due to hepatic toxicity when serum hepatic enzyme levels exceeded $300 \mathrm{U} / \mathrm{L}$; five-fold the normal levels ${ }^{16,17}$. In case of suspicion for salicylate intoxication serum concentration of ASA was also examined.

This study was approved by Ethics Committee of Hospital (Ref no: 2014-017). The informed consent mentioning current treatment approaches was obtained from parents of all patients, before the start of therapy.

\section{Statistical Analysis}

The therapy results and adverse events in each group were compared using SPSS for Windows software package v.17.0 (SPSS, Chicago, IL, USA). The Kolmogorov-Smirnov test was used for the assessment of homogeneity of variables. The data were analyzed for comparison of 2 groups using the Mann-Whitney U-test and for more than 2 groups using the KruskalWallis test. Statistical significance was defined as $\mathrm{P}<0.05$.

\section{Results}

The study cohort consisted of 338 patients with ARF after exclusion of 13 patients diagnosed with FMF, JIA or HSP and 18 patients in whom ASA was replaced with NXN due to hepatic toxicity during follow-up (Fig. 1). The female/male ratio was $1.11(178 / 160)$, mean age of patients was $10.3 \pm 2.2(5-18)$ years. The median follow-up was 62 (3-125) months. There was fever at presentation in $28.9 \%$ of patients. Monoarthritis was identified in $18.6 \%$ of patients, all with valvular involvement. Silent carditis was present in $20.1 \%$ of patients.

Table I shows patient demographics of 4 treatment subgroups. There were 122 patients $(36.1 \%)$ in group $1 \mathrm{~A}, 111$ patients $(32.8 \%)$ in group $1 \mathrm{~B}, 58$ patients $(17.2 \%)$ in group $2 \mathrm{~A}$ and 47 patients $(13.9 \%)$ in group $2 \mathrm{~B}$. There were no significant differences for age and gender among the groups.

Table II shows the clinical and laboratory data of patients. Fever resolved in 1 day and arthritis or arthralgia resolved in 2 days in all groups. APR normalized median in 1 (1-4) week in patients given steroids and in 2 (1-7) weeks in patients given NXN or ASA. There were no significant differences between groups with regard to ESR and CRP concentrations. On admission, the mean values of ESR and CRP were $80.2 \pm 20.7(41-150) \mathrm{mm} / \mathrm{h}$ and $7.3 \pm 5.4(0.8-28) \mathrm{mg} / \mathrm{dl}$. The cut-off point is $20 \mathrm{~mm} / \mathrm{h}$ for ESR and $0.4 \mathrm{mg} / \mathrm{dl}$ for CRP. Sixteen patients $(13.1 \%)$ in group $1 \mathrm{~A}$ and 13 patients $(11.7 \%)$ in group $1 \mathrm{~B}$ showed worsening or new valvular regurgitations, regressing to pre-treatment levels or getting better during follow-up.

The adverse events were more frequent in patients receiving ASA. In group 1, the gastric adverse effects resistant to single gastroprotective medicine (a proton-pump inhibitor) were seen in 16 patients given ASA versus in 4 patients given NXN $(\mathrm{p}<0.05)$. Excluding 3 patients with rash and headache, no other adverse effects were observed with NXN $(\mathrm{p}<0.05)$. Neither treatment was changed nor any patient was excluded from study because of gastric adverse effects. Instead, a second or third gastro-protective medicine $\left(\mathrm{a} \mathrm{H}_{2}\right.$ receptor blocker or an antacid) was instituted to overcome these adverse effects. In group 2, the adverse reactions were almost similar and mostly attributed to steroid usage. Salicylate intoxication, Reye syndrome, jaundice, chronic hepatic toxicity, renal toxicity or coagulation defects were not observed in any patient.

Table III shows the treatment outcome. Rebound was observed in $28(8.3 \%)$ and recurrence was detected in $42(12.4 \%)$ patients. The rate of compliance to prophylaxis with benzathine penicillin $\mathrm{G}$ was $82.8 \%$. There was a significant difference in recurrence rate between patients, compliant or non-compliant to benzathine penicillin $\mathrm{G}$ prophylaxis $(8 / 34,23.5 \%, \mathrm{p}<0.01)$. The rate of rebound, recurrence and the prevalence of RVD were not different in patients given NXN, ASA or mPSL. The RVD persisted in 56 patients $(45.9 \%)$ in group $1 \mathrm{~A}$, in 44 patients $(39.6 \%)$ in group $1 \mathrm{~B}$, in 28 patients $(48.3 \%)$ in group $2 \mathrm{~A}$ and in 22 patients $(46.8 \%)$ in group 2B. Sixty-five patients (19.2\%) presented later on with chorea. 


\section{Discussion}

There are two major issues in this era that we have to discuss nowadays. Firstly, we are in need of new definitions for diagnostic criteria, because the revised Jones criteria work only in typical cases ${ }^{11}$. Secondly, new therapeutic strategies for treatment of ARF are also needed, because there are lots of adverse effects with drugs used and there is no drug showing superior efficacy and reducing the risk of RVD ${ }^{3}$.

No significant changes in the diagnosis of ARF have been observed since early 1940's to the present $^{18,19}$. However, in some countries with high incidence of ARF and high prevalence of RVD, something has to be changed because of the diversity of symptoms and findings. As a result, in Australia polyarthralgia, aseptic monoarthritis and subclinical carditis are accepted as major manifestations of disease in high risk areas ${ }^{20,21}$. Similarly, The New Zealand guideline has accepted subclinical carditis and monoarthritis associated with antiinflammatory drug usage as major criteria of $\mathrm{RF}^{22}$. According to a recent report, utilisation of the New Zealand guideline resulted in $16 \%$ more cases defined as definite ARF than using revised Jones criteria. Subclinical carditis was present in $30 \%$ and monoarthritis with antiinflammatory drug usage was present in $11 \%^{23}$. According to them, this guideline resulted in improved consistency in the approach reducing morbidity and mortality from this disease. So, subclinical carditis is not a benign condition and echocardiography should be used as a criterion in the diagnosis 24,25 . Thus, new definitions in diagnostic criteria have created new insights. Finally, the AHA has recently published a revision to the Jones Criteria for the diagnosis of ARF in the era of Doppler echocardiography ${ }^{11}$.

As there are no significant changes in the management of ARF for years, we are in need of new strategies for treatment ${ }^{1,3,26}$. So far, there are 2 major drugs used in the management of ARF; steroids and ASA. However, both types of drug have various adverse effects. Weight gain, moon face, acne formation, hypertension and hyperglycemia are well-known adverse effects of steroids ${ }^{27}$. Hepatic toxicity, gastric pain and discomfort, rash, dizziness and headache are frequent adverse events during ASA treatment. Hepatic transaminase elevation is seen in about $25 \%$ of patients under ASA treatment with plasma levels exceeding 10 $\mathrm{mg} / \mathrm{dl}^{16}, 17,28$. Salicylate intoxication, Reye syndrome, jaundice, chronic hepatic toxicity, renal toxicity or coagulation defects may also be seen in these patients ${ }^{29}$. Inspite of all these adverse effects and the requirement of close laboratory follow-up, few studies with NSAIDs other than ASA have been performed in the management of $\mathrm{ARF}^{1,3}$.

In this study, the rates of improvement in laboratory and clinical parameters in ASA and NXN groups were similar. Gastric adverse effects were more common in ASA group than in NXN group, and ASA was changed to NXN in $10.2 \%$ of patients due to hepatic toxicity. So, this study demonstrated that NXN is effective in ARF with or without carditis, and it is well tolerated. Therefore, NXN is a safe and effective alternative to ASA for treatment of ARF.

Although, good results were reported with these new NSAIDs, such as naproxen and tolmetin $^{7-9}$, it is not clear, why they have not come into routine use in the treatment of ARF. For example, Uziel et al. ${ }^{7}$ showed that NXN may be effective and safe for treatment of arthritis and fever in a retrospective series of 19 patients with ARF, but there was no control group. Later, Hashkes et al. ${ }^{8}$ conducted a randomised-controlled study in 33 patients and found NXN to be equivalent to ASA for treatment of arthritis and less likely to cause hepatic toxicity. In a study comparing the effect of tolmetin versus ASA in 72 patients with ARF, Karademir et al. ${ }^{9}$ showed that tolmetin was safe and effective in patients with ARF without carditis.

Apart from showing similar results, the present study has preciously showed these results in a large study cohort of 338 patients and also in patients with arthritis and carditis.

Several NSAIDs have been used successfully in other pediatric rheumatic diseases with almost equal efficacy and tolerance for years. Moreover, similar efficacy of NXN to that of ASA in JIA and reactive arthritis has been reported previously $5,30,31$. It is important to consider susceptibility to toxicity in treating children while ensuring normal growth and development ${ }^{6}$. Additionally, the iatrogenic costs related to drug-induced adverse events are a significant component of the total cost 
of therapy in rheumatic diseases ${ }^{32}$. While, the cost of both drugs is similar, about $\$ 5$ $(€ 4.5)$ for 1 month, for a $27-\mathrm{kg}$ child, in our country, NXN has some advantages over ASA, including the following: less frequent dosing, good patient compliance, not being associated with Reye syndrome and uncommon hepatic toxicity ${ }^{29}$. ASA requires close monitoring because of intoxication risk, hepatic toxicity or Reye syndrome.

In conclusion, the two major drugs used in the treatment of ARF, steroids and ASA, have various adverse effects. We are in need of new studies addressing other anti-inflammatory agents to establish an evidence for the efficacy of these drugs with possibly less side effects. NXN has some advantages over ASA and as shown in the present study, it is a safe and effective alternative to ASA in the treatment of ARF.

There are several limitations in this study. First of all, this is not a randomized and blinded study. This study was planned in a retrospective manner, because we wanted to see the initial results with NXN in patients with isolated arthritis. The frequency of NXN prescription has been increased concurrently with good results over the last 7 years. After a while, the patients with carditis have been included in the treatment with NXN. So, there is a possibility of selection bias or treatment allocation bias. Secondly, the data were reviewed using a database system including routine charts prepared for each patient. Nonetheless, this may have caused a recall bias or information bias during an 11-year period. In light of the foregoing, to establish and confirm the efficacy and safety of NXN in the treatment of ARF, prospective, randomized and controlled trials are required.

\section{REFERENCES}

1. Webb RH, Grant C, Harnden A. Acute rheumatic fever. BMJ 2015; 351: h3443.

2. Orün UA, Ceylan O, Bilici M, et al. Acute rheumatic fever in the central Anatolia Region of Turkey: A 30year experience in a single center. Eur J Pediatr 2012; 171: 361-368.

3. Cilliers A, Adler AJ, Saloojee H. Anti-inflammatory treatment for carditis in acute rheumatic fever. Cochrane Database Syst Rev 2015; 28: CD003176.

4. Southwood TR. Arthritis in children. BMJ 1995; 310: 728-732.
5. Hollingworth P. The use of non-steroidal antiinflammatory drugs in paediatric rheumatic diseases. Br J Rheumatol 1993; 32: 73-77.

6. Milojevic DS, Ilowite NT. Treatment of rheumatic diseases in children: special considerations. Rheum Dis Clin North Am 2002; 28: 461-482.

7. Uziel Y, Hashkes PJ, Kassem E, Padeh S, Goldman $\mathrm{R}$, Wolach $\mathrm{B}$. The use of naproxen in the treatment of children with rheumatic fever. J Pediatr 2000; 137: 269-271.

8. Hashkes PJ, Tauber T, Somekh E, et al; Pediatric Rheumatology Study Group of Israel. Naproxen as an alternative to aspirin for the treatment of arthritis of rheumatic fever: a randomized trial. J Pediatr 2003; 143: 399-401.

9. Karademir S, Oğuz D, Șenocak F, Öcal B, Karakurt C, Çabuk F. Tolmetin and salicylate therapy in acute rheumatic fever: Comparison of clinical efficacy and side effects. Pediatr Int 2003; 45: 676-679.

10. The Special Writing Group of the Committee on Rheumatic Fever, Endocarditis and Kawasaki Disease of the Council on Cardiovascular Disease in the Young of the American Heart Association. Guidelines for the diagnosis of rheumatic fever: Jones criteria, 1992 update. JAMA 1992; 268: 2069-2073.

11. American Heart Association Committee on Rheumatic Fever, Endocarditis, and Kawasaki Disease of the Council on Cardiovascular Disease in the Young. Revision of the Jones Criteria for the diagnosis of acute rheumatic fever in the era of Doppler echocardiography: a scientific statement from the American Heart Association. Circulation 2015; 131: 1806-1818.

12. Lai WW, Geva T, Shirali GS, et al; Task Force of the Pediatric Council of the American Society of Echocardiography; Pediatric Council of the American Society of Echocardiography. Guidelines and standarts for performance of a pediatric echocardiogram: a report from the Task Force of the Pediatric Council of the American Society of Echocardiography. J Am Soc Echocardiogr 2006; 19: 1413-1430.

13. ACC/AHA Task Force on Practice Guidelines. 2008 focused update incorporated into the ACC/AHA 2006 guidelines for the management of patients with valvular heart disease. J Am Coll Cardiol 2008; 52: e1-142.

14. Working Group on Pediatric Acute Rheumatic Fever and Cardiology Chapter of Indian Academy of Pediatrics, Saxena A, Kumar RK, Gera RP, Radhakrishnan S, Mishra $\mathrm{S}$, Ahmed Z. Consensus guidelines on pediatric acute rheumatic fever and rheumatic heart disease. Indian Pediatr 2008; 45: 565-573.

15. Punukollu M, Mushet N, Linney M, Hennessy C, Morton M. Neuropsychiatric manifestations of Sydenham's chorea: a systematic review. Dev Med Child Neurol 2016; 58: 16-28.

16. Zimmermann HJ. Effects of aspirin and acetaminophen on the liver: Arch Intern Med 1981; 141: 333-342.

17. Benson GD. Hepatotoxicity following the therapeutic use of antipiretic analjesics. Am J Med 1983; 75: 8593. 
18. Ferrieri P; Jones Criteria Working Group. Proceedings of the Jones Criteria workshop. Circulation 2002; 106: 2521-2523.

19. Burke RJ, Chang C. Diagnostic criteria of acute rheumatic fever. Autoimmun Rev 2014; 13: 503-507.

20. Carapetis JR, Brown A, Wilson NJ, Edwards KN. An Australian guideline for rheumatic fever and rheumatic heart disease. Med J Aust 2007; 186: 581-586.

21. Smith MT, Zurynski Y, Lester-Smith D, Elliott E, Carapetis J. Rheumatic fever: Identification, management and secondary prevention. Aust Fam Physician 2012; 41: $31-35$.

22. Atatoa-Carr P, Lennon D, Wilson N. Rheumatic fever diagnosis, management and secondary prevention: a New Zealand guideline. NZ Med J 2008; 121: 59-69.

23. Wilson NJ, Voss L, Morreau J, Stewart J, Lennon D. New Zealand guidelines for the diagnosis of acute rheumatic fever: small increase in the incidence of definite cases compared to the American Heart Association Jones criteria. NZ Med J 2013; 126: 50-59.

24. Şahin M, Yıldırım I, Özkutlu S, Alehan D, Özer S, Karagöz T. Clinical features and mid- and long-term outcomes of pediatric patients with subclinical carditis. Turk J Pediatr 2012; 54: 486-492.

25. Pekpak E, Atalay S, Karadeniz C, Demir F, Tutar E, Uçar T. Rheumatic silent carditis: Echocardiographic diagnosis and prognosis of long-term follow-up. Pediatr Int 2013; 55: 685-689.
26. Gerber MA, Baltimore RS, Eaton CB, et al. Prevention of rheumatic fever and diagnosis and treatment of acute Streptococcal pharyngitis: a scientific statement from the American Heart Association Rheumatic Fever, Endocarditis, and Kawasaki Disease Committee of the Council on Cardiovascular Disease in the Young, the Interdisciplinary Council on Functional Genomics and Translational Biology, and the Interdisciplinary Council on Quality of Care and Outcome Research: endorsed by the American Academy of Pediatrics. Circulation 2009; 119: 1541-1551.

27. Frauman AG. An overview of the adverse reactions to adrenal corticosteroids. Adv Drug React Toxicol Rev 1996; 15: 203-206.

28. Aithal GP, Day CP. Nonsteroidal anti-inflammatory drug-induced hepatotoxicity. Clin Liver Dis 2007; 11: 563-575.

29. Brune K, Patriqnani P. New insights in to the use of currently available non-steroidal anti-inflammatory drugs. J Pain Res 2015; 8: 105-118.

30. Lindsley CB. Uses of nonsteroidal anti-inflammatory drugs in pediatrics. Am J Dis Child 1993; 147: 229236.

31. Litalien C, Jacqz-Aigrain E. Risks and benefits of nonsteroidal anti-inflammatory drugs in children. Paediatr Drugs 2001; 3: 817-858.

32. Hunsche E, Chancellor JV, Bruce N. The burden of arthritis and nonsteroidal anti-inflammatory treatment. Pharmacoeconomics 2001; 19 (Suppl 1): 1-15. 